\title{
Anälise da vulnerabilidade entre estudantes da rede pública e privada
}

\author{
Analysis of vulnerability among students in the public \\ and private network
}

Lediana Dalla Costa ${ }^{1}$, Daniela Tavares Camera ${ }^{2}$, Ana Maria Zeferino ${ }^{3}$, Anderson Kalinoski ${ }^{4}$, Marcela Gonçalves Trevisan ${ }^{5}$, Franciele do Nascimento Santos Zonta ${ }^{6}$

\begin{abstract}
1. Enfermeira. Mestre em Saúde e Gestão do Trabalho. Docente e Coordenadora do Curso de Graduação em Enfermagem da Universidade Paranaense (UNIPAR). Francisco Beltrão - PR - Brasil. E-mail: lediana@prof.unipar.br ORCID: https://orcid.org/0000-0002-9114-3669

2. Graduanda em Enfermagem pela Universidade Paranaense (UNIPAR). Francisco Beltrão - PR - Brasil. E-mail: daniela.camera@edu.unipar.br ORCID: https://orcid.org/0000-0001-8018-8629

3. Enfermeira. Graduada em Enfermagem pela Universidade Paranaense (UNIPAR). Francisco Beltrão - PR - Brasil. E-mail: ana.zeferino@edu.unipar.br ORCID: https://orcid.org/0000-0001-7726-6905

4. Enfermeiro. Graduado em Enfermagem pela Universidade Paranaense (UNIPAR). Francisco Beltrão PR - Brasil. E-mail: anderson.k@edu.unipar.br ORCID: https://orcid.org/0000-0001-7160-6753

5. Enfermeira. Especialista em Saúde Pública com Ênfase na Atenção à Saúde da Mulher. Responsável Técnica do Estágio Curricular Obrigatório de Saúde Pública do Curso de Graduação em Enfermagem da Universidade Paranaense (UNIPAR). Francisco Beltrão - PR - Brasil. E-mail: marcelatrevisan@unipar. br ORCID: https://orcid.org/0000-0002-1703-7200

6. Enfermeira. Mestre em Ciência da Saúde. Docente do Curso de Graduação em Enfermagem da Universidade Paranaense (UNIPAR). Francisco Beltrão - PR - Brasil. E-mail: franciele.ns@prof.unipar.br ORCID: https://orcid.org/0000-0002-4236-4027
\end{abstract}

CONTATO: Lediana Dalla Costa | Endereço: Avenida Júlio Assis Cavalheiro, 2000 - Industrial | CEP 85.601-000 | Francisco Beltrão | Paraná | Brasil | Telefone: (46) 3520-2800 | E-mail: lediana@prof.unipar.br

COMO CITAR: Costa LD, Camera DT, Zeferino AM, Kalinoski A, Trevisan MG, Zonta FNS. Análise da vulnerabilidade entre estudantes da rede pública e privada. R. Saúde Públ. Paraná. 2020 Jul;3(1):108-119. 
RESUMO A adolescência é caracterizada como a passagem da infância para a fase adulta. Período de maior suscetibilidade a situações de risco. Assim, objetivou-se analisar e comparar as vulnerabilidades de estudantes do ensino médio público e privado em um município paranaense. Trata-se de uma pesquisa de campo, transversal, exploratória-descritiva, de caráter quantitativo, realizada por meio da aplicação de um questionário fechado adaptado. A pesquisa foi constituída por 470 estudantes, sendo 351 da rede pública e 119 da privada. Observouse que, alguns já fizeram uso de cigarro e drogas ilícitas, enquanto a maioria já consumiu bebida alcoólica. Acerca do pensamento e da tentativa de suicídio, os alunos de escolas privadas são mais propensos. Ainda, evidenciou-se que, os alunos de escolas públicas apresentaram maior vulnerabilidade. Nesse contexto, salienta-se a importância da promoção e prevenção de saúde por intermédio de ações de educação em saúde nas escolas, voltadas para essa população.

PALAVRAS-CHAVE: Análise de Vulnerabilidade. Adolescentes. Sexualidade.

ABSTRACT Adolescence is the passage from childhood to adulthood. This is a period when adolescents are most susceptible to risky situations. Thus, the objective of this study was to analyze and compare the vulnerabilities of public and private high school students in a municipality of the state of Paraná. This is a quantitative, cross-sectional, exploratory-descriptive field research using a closed, adapted questionnaire. The research consisted of 470 students, 351 from the public, and 119 from the private schools. It was observed that some have already used cigarettes and illicit drugs, while most of them have consumed alcohol. Private school students are more likely to think of and attempt suicide, and public school students show more vulnerability. The importance of health promotion and prevention through health education actions in schools aimed at this population is emphasized.

KEYWORDS: Vulnerability Analysis. Adolescent. Sexuality.

\section{INTRODUÇÃO}

A

adolescência é considerada um período de inúmeras mudanças físicas, psíquicas, emocionais e sociais, que alteram os padrões de comportamentos. De acordo com a Organização Mundial da Saúde (OMS), a adolescência abrange dos 10 aos 19 anos de idade. Contudo, o Estatuto da Criança e do Adolescente (ECA) considera, pela Lei no 8.069 de 1990, a faixa etária entre 12 e 18 anos de idade?. É nessa etapa que ocorre a descoberta de sua sexualidade, o conhecimento de seu corpo, busca por prazer e novas experiências².

Cerca de 30,3\% da população brasileira encontra-se na faixa etária dos 10 aos 24 anos, o que representa cerca de 54 milhões de adolescentes e jovens, sendo que são esses indivíduos com maior exposição a riscos 
relacionados à vulnerabilidade, entre eles o tabagismo, etilismo, uso de drogas ilícitas, comportamento sexual de risco e diminuição dos cuidados com a saúde³.

De acordo com uma estimativa elaborada pelo Centro Brasileiro de Informações sobre Drogas Psicotrópicas (CEBRID), reafirmada pela OMS, aproximadamente 90\% dos fumantes torna-se dependente da nicotina entre 5 e 19 anos de idade, o que corresponde a cerca de 30 milhões de indivíduos no País. Já em relação ao álcool, verificou-se uma associação entre o uso de álcool e a não adesão a métodos contraceptivos em adolescentes de 13 a 15 anos, o que contribui para maior vulnerabilidade às Infecções Sexualmente Transmissiveis (IST) e à gravidez não planejada4.

Outro fator relevante refere-se ao início da vida sexual, devido à sua ocorrência precoce entre os adolescentes. De acordo com dados da Pesquisa Nacional de Saúde do Escolar (PeNSE) de 2015, 27,5\% dos adolescentes na faixa etária de 13 a 15 anos já iniciaram sua vida sexual e segundo o Ministério da Saúde (MS), a média da idade da iniciação sexual tem sido de 14,9 anos ${ }^{5-6}$.

O início da atividade sexual por pessoas muito jovens é considerado fator de risco para IST e gravidez precoce. Conforme dados do MS, os casos de Síndrome da Imunodeficiência Adquirida (AIDS) entre jovens de 15 a 24 anos foi de 11,8 por 100 mil habitantes no ano de 2012 .

Faz-se necessário uma atenção à sexualidade dos adolescentes com o intuito de contribuir para a redução dos problemas relacionados à sua vida pessoal e social. A escola tem um importante papel em sua educação sexual, visto que é um ambiente apropriado para a aprendizagem da anatomia e fisiologia do corpo humano e também sobre métodos de prevenção de gravidez não planejada e IST ${ }^{8}$.

Nesse aspecto, os profissionais da área da saúde e da educação precisam informar aos pais sobre a importância de um maior contato com seus filhos nessa fase da vida, destacando o valor de uma boa convivência familiar? .

Através de estudos feitos no Brasil, a desigualdade socioeconômica é um dos fatores que mais propicia a vulnerabilidade para IST, o uso de substâncias psicoativas, o suicídio e até mesmo a gestação precoce. Ela está presente em todas as fases da vida humana, mas principalmente na adolescência. Dessa forma, com a carência de políticas públicas voltadas aos adolescentes, observa-se um grande aumento de situações de risco e vulnerabilidade, necessitando de ações específicas para o seu enfrentamento ${ }^{10}$.

Durante a formação do adolescente, a escola tem um importante papel no desenvolvimento cognitivo, emocional e social. Logo, cabe às instituições de ensino, em paralelo com os serviços de saúde, instituírem estratégias que possibilitem o monitoramento dos fatores e situações de risco para proteção desses indivíduos.

Diante dessa problemática, é possível observar diferenças entre os fatores de vulnerabilidade de estudantes de escolas públicas e privadas? Nesse contexto, traçou-se como objetivo avaliar os fatores de vulnerabilidade de estudantes do ensino médio de escolas públicas e privadas do município de Francisco Beltrão, estado do Paraná.

\section{MÉTODO}

Configura-se como uma pesquisa descritiva, de caráter transversal e exploratório, com abordagem quantitativa, desenvolvida nas escolas públicas e privadas de ensino médio de um município do sudoeste do Paraná. 
A pesquisa foi realizada em 14 escolas públicas e 3 escolas privadas de ensino médio no ano de 2018. Para o cálculo amostral utilizou-se equação para estimativa de tamanho populacional a partir de 1.415 estudantes matriculados no ensino médio, no qual inserimos uma prevalência de vulnerabilidade de 50\%, tendo em vista a grande variedade de prevalências descritas na literatura. No cálculo final foram acrescidos 10\% no número amostral ( $\mathrm{n}=303$ ) para para suprir as possíveis perdas e recusa, resultando em um número mínimo de 333 estudantes.

Participaram do estudo os alunos matriculados no ensino médio nos períodos matutino, vespertino, noturno ou integral de escolas públicas e privadas, que apresentaram o consentimento dos pais e/ou responsáveis por meio da assinatura do Termo de Consentimento Livre e Esclarecido (TCLE) e que estavam presentes na sala de aula no momento da coleta de dados.

A coleta de dados foi realizada entre os períodos de maio a julho nas escolas públicas e de abril a setembro nas escolas privadas e deu-se em dois momentos. Primeiramente foi solicitada a autorização para os pais e/ou responsáveis por meio da entrega do TCLE aos alunos. O segundo destinou-se ao recolhimento das autorizações e à aplicação do questionário.

Trata-se de uma adaptação do instrumento Juventude Brasileira-Versão Fase II"1 que abrange perguntas fechadas relacionadas à idade, sexo, escolaridade, características sociodemográficas e econômicas, uso de substâncias psicoativas, relação sexual, conhecimento sobre infecções sexualmente transmissíveis, gravidez precoce, comportamento suicida e utilização de métodos contraceptivos.

Após autorização da Secretaria Municipal de Educação, seguindo todos os preceitos éticos e legais da Resolução n 466/2012 do Conselho Nacional de Saúde (CNS), a pesquisa foi submetida ao Comitê de Ética em Pesquisa Envolvendo Seres Humanos (CEPEH) da Universidade Paranaense (UNIPAR) por meio de dois projetos de pesquisa. Um direcionava-se aos alunos de escolas públicas e outro a escolas privadas.

Os projetos foram aprovados sob os protocolos $n^{0} 2.596 .383$ e $n^{0} 2.562 .912$, respectivamente. Os dados foram tabulados e analisados por meio do software Statistical Package for Social Science ${ }^{\circledR}$ (SPSS) versão 25.0, para avaliar frequências absolutas e relativas e, posteriormente, foram correlacionados através do teste qui-quadrado, sendo considerado significativos $p<0,05$.

\section{RESULTADOS}

A pesquisa foi constituída por 470 estudantes, sendo 351 adolescentes de escolas públicas e 119 adolescentes de escolas privadas. Essa proporção justifica-se em razão das escolas privadas disporem de um menor número de matrículas.

Considerando o sexo dos estudantes, constatou-se que a maioria dos participantes de ambas as redes de ensino eram do sexo feminino, 70 (23,7\%) de escolas privadas e 224 (76,3\%) de públicas. Em relação à religiosidade, a maioria dos estudantes da escola pública e privada seguia alguma religião e não tinha filhos, totalizando, respectivamente, 382 e 467 alunos. Quanto ao risco familiar, notou-se que 293 adolescentes moravam com os pais e 178 moravam apenas com um dos pais.

Quanto ao uso do cigarro, 95 estudantes já fumaram pelo menos 1 cigarro. Em relação ao consumo de álcool, a maioria de ambas as escolas já consumiram, como mostra a Tabela 1.

Ainda na Tabela 1, fica evidenciado o uso de drogas ilícitas, sendo que 56 estudantes já consumiram alguma vez esse tipo de substância. 
Tabela 1 - Dados sociodemográficos e a frequência do uso de álcool e drogas ilícitas por adolescentes estudantes de escolas públicas e privadas de um município paranaense no ano de 2018. Paraná - Brasil.

\begin{tabular}{|c|c|c|c|c|}
\hline \multirow{2}{*}{ VARIÁVEIS } & \multicolumn{2}{|c|}{ ESCOLA } & \multirow{2}{*}{ TOTAL } & \\
\hline & Privada & Pública & & \\
\hline SEXO & n (\%) & n (\%) & & \\
\hline Feminino & $70(23,7)$ & $224(76,3)$ & $294(100,0)$ & \\
\hline Masculino & $49(27,8)$ & $127(72,2)$ & $176(100,0)$ & \\
\hline \multicolumn{5}{|l|}{ SEGUE ALGUMA RELIGIÃO } \\
\hline $\operatorname{Sim}$ & $97(25,4)$ & $285(74,6)$ & $382(100,0)$ & \\
\hline Não & $22(24,7)$ & $67(75,3)$ & $89(100,0)$ & \\
\hline \multicolumn{5}{|l|}{ TEM FILHOS } \\
\hline $\operatorname{Sim}$ & $1(25,0)$ & $3(75,0)$ & $4(100,0)$ & \\
\hline Não & $118(25,3)$ & $349(74,7)$ & $467(100,0)$ & \\
\hline \multicolumn{5}{|l|}{ RISCO FAMILIAR } \\
\hline Mora com os pais & $85(29,0)^{\star}$ & $208(71,0)$ & $293(100,0)$ & 0,01 \\
\hline Mora com apenas um dos pais & $34(19,1)$ & $144(80,9)^{\star}$ & $178(100,0)$ & 0,02 \\
\hline \multicolumn{5}{|l|}{ USO DE CIGARRO } \\
\hline $\operatorname{Sim}$ & $30(31,6)$ & $65(68,4)$ & $95(100,0)$ & \\
\hline Não & $89(23,7)$ & $287(76.3)$ & $376(100,0)$ & \\
\hline \multicolumn{5}{|l|}{ USO DE BEBIDA ALCOÓLICA } \\
\hline Sim & $102(26,6)$ & $281(73,4)$ & $383(100,0)$ & \\
\hline Não & $17(19,3)$ & $71(80,7)$ & $88(100,0)$ & \\
\hline \multicolumn{5}{|l|}{ USOU DROGA ILÍCITA } \\
\hline Sim & $14(25,0)$ & $42(75,0)$ & $56(100,0)$ & \\
\hline Não & $105(25,3)$ & $310(74,7)$ & $415(100,0)$ & \\
\hline
\end{tabular}

Fonte: Dados da pesquisa, 2018.

Na Tabela 2, estão descritas as associações entre o pensamento e a tentativa de suicídio, sendo evidenciado que alunos de escolas privadas são mais propensos ao pensamento suicida. Entretanto, em relação à tentativa, a escola pública apresenta maiores índices.

Tabela 2 - Índice de pensamento e tentativa de suicídio entre adolescentes estudantes de escolas públicas e privadas de um município paranaense no ano de 2018. Paraná - Brasil.

\begin{tabular}{lcccccccc}
\hline & \multicolumn{1}{c}{ ESCOLA PRIVADA } & \multicolumn{2}{c}{ ESCOLA PÚBLICA } & \multicolumn{2}{c}{ TOTAL } & P \\
\hline PENSAMENTO SUICIDA & $\mathbf{n}$ & $\%$ & $\mathbf{n}$ & $\%$ & $\mathbf{n}$ & $\%$ & \\
Sim & 47 & $(39,5)^{\star}$ & 112 & $(31,85)$ & 159 & $(100,0)$ & 0,01 \\
Não & 72 & $(23,1)$ & 240 & $(76,9)$ & 312 & $(100,0)$ & \\
TENTATIVA DE SUICÍDIO & & & & & & & \\
Sim & 28 & $(41,8)$ & 39 & $(58,2)^{\star}$ & 67 & $(100,0)$ & 0,001 \\
Não & 91 & $(22,5)$ & 313 & $(77,5)$ & 404 & $(100,0)$ & \\
\hline
\end{tabular}


Com relação ao comportamento sexual desses adolescentes, a Tabela 3 indica que a maioria ainda não teve a primeira relação sexual. Contudo, 190 estudantes já iniciaram sua vida sexual, sendo um número significativo por ser uma faixa etária inferior a 18 anos. Quanto ao uso de camisinha na primeira e última relação, evidenciouse que alguns adolescentes referiram não usar camisinha como forma de prevenção de IST e gravidez, apesar da maioria ter demonstrado conhecimento sobre métodos contraceptivos. Aos que já iniciaram sua vida sexual, grande parte possuía parceiro fixo, sendo um fator de proteção para IST.

Tabela 3 - Comportamento sexual e conhecimento de métodos contraceptivos entre adolescentes de ensino médio de escolas públicas e privadas de um município paranaense no ano de 2018. Paraná, Brasil. 2019.

\begin{tabular}{|c|c|c|c|c|c|}
\hline \multirow[b]{3}{*}{ JÁ TEVE RELAÇÃO SEXUAL } & \multicolumn{3}{|c|}{ ESCOLA } & \multirow{2}{*}{ TOTAL } & \multirow{3}{*}{$\mathbf{P}$} \\
\hline & \multicolumn{2}{|c|}{ Privada } & Pública & & \\
\hline & $\mathrm{n}$ & $\%$ & $\%$ & $\%$ & \\
\hline $\operatorname{Sim}$ & 45 & $(23,7)$ & $145(76,3)^{*}$ & $190(100,0)$ & 0,02 \\
\hline Não & 74 & $(26,3)$ & $207 \quad(73,7)$ & $281(100,0)$ & \\
\hline \multicolumn{6}{|c|}{ USO DE PRESERVATIVO NA $1^{\text {a }}$ RELAÇÃO SEXUAL } \\
\hline $\operatorname{Sim}$ & 35 & $(24,5)$ & $108 \quad(75,5)$ & $143(100,0)$ & 0,05 \\
\hline Não & 10 & $(21,3)$ & $37^{\star} \quad(78,7)$ & $47 \quad(100,0)$ & \\
\hline \multicolumn{6}{|c|}{ USO DE CAMISINHA NA ÚLTIMA RELAÇÃO } \\
\hline $\operatorname{Sim}$ & 35 & $(27,8)$ & $91 \quad(72,2)$ & $126(100,0)$ & \\
\hline Não & 10 & $(15,6)$ & $54 \quad(84,4)$ & $64 \quad(100,0)$ & \\
\hline \multicolumn{6}{|l|}{ JÁ CONTRAIU IST } \\
\hline $\operatorname{Sim}$ & 0 & $(0,0)$ & $2(100,0)$ & $2(100,0)$ & \\
\hline Não & 119 & $(25,4)$ & $350(74,6)$ & $469(100,0)$ & \\
\hline \multicolumn{6}{|l|}{ POSSUI PARCEIRO FIXO } \\
\hline Sim & 29 & $(20,4)$ & $113(79,6)$ & $142(100,0)$ & \\
\hline Não & 16 & $(33,3)$ & $32(66,7)$ & $48 \quad(100,0)$ & \\
\hline Não se aplica & 74 & $(26,3)$ & $207 \quad(73,7)$ & $281(100,0)$ & \\
\hline \multicolumn{6}{|c|}{ CONHECE MÉTODOS CONTRACEPTIVOS } \\
\hline $\operatorname{Sim}$ & 117 & $(29,2)$ & $284(70,8)$ & $401(100,0)$ & 0,000 \\
\hline Não & 2 & $(2,9)$ & $68(97,1)^{\star}$ & $70(100,0)$ & \\
\hline
\end{tabular}

Fonte: Dados da pesquisa, 2018

Em relação às vulnerabilidades, identificou-se uma média de 6,231 vulnerabilidades (DP + ou - 2,49). Entretanto, foi possível verificar que os adolescentes apresentaram no mínimo uma vulnerabilidade e, no máximo, 15. Observou-se que alunos de escolas públicas apresentaram maior vulnerabilidade em relação aos alunos de escolas privadas. Porém, 100\% dos alunos da rede privada revelaram 5 ou mais vulnerabilidades. 
Tabela 4 - Quantitativo de vulnerabilidades entre adolescentes estudantes de escolas públicas e privadas de um município paranaense no ano de 2018. Paraná - Brasil. 2019.

\begin{tabular}{lccccc}
\hline \multirow{2}{*}{ VULNERABILIDADES } & \multicolumn{2}{c}{ ESCOLA } & \multirow{2}{*}{ TOTAL } & P \\
\cline { 2 - 3 } & Pública & Privada & & \\
\hline 1 a 5 vulnerabilidades & $198(56,3)$ & $0(0,0 \%)$ & & $42,0 \%$ & \\
$\mathbf{5}$ ou mais & $154(56,4)^{\star}$ & $119(43,6)$ & & $58,0 \%$ & 0,000 \\
\hline
\end{tabular}

Fonte: Dados da pesquisa, 2018.

\section{DISCUSSÃo}

A adolescência caracteriza-se por um período de intensas mudanças físicas, cognitivas e sociais, o que torna os indivíduos desta faixa etária suscetíveis a diversas vulnerabilidades, caracterizadas por um conjunto de fatores sociais, culturais e epidemiológicos, que podem aumentar ou diminuir os fatores de riscos e de proteção, entre eles o uso de drogas lícitas e ilícitas, vida sexual precoce e desprotegida².

A pesquisa evidenciou que existem fatores de risco em ambas as escolas. Todavia, algumas variáveis apresentaram significância quando comparadas às escolas públicas ou privadas. Sem dúvida, entender as peculiaridades desta população pode contribuir para uma melhor estratégia de prevenção e promoção de saúde.

No que tange ao sexo dos participantes, observou-se que houve predominância do feminino, dados que se assemelham ao estudo realizado por pesquisadores brasileiros, no qual o sexo feminino também prevaleceu ${ }^{13}$. O Instituto Brasileiro de Geografia e Estatística (IBGE), em 2019, por meio da Pesquisa Nacional por Amostra de Domicílios Contínua (PNAD), revelou que o número de mulheres no Brasil é superior ao de homens. A população brasileira é composta por $48,3 \%$ de homens e $51,7 \%$ de mulheres, resultando no maior número de indivíduos do sexo feminino matriculados nas escolas ${ }^{14}$.

No que concerne à religião, observou-se que a maioria dos estudantes referiu seguir uma religião, o que difere do estudo realizado por pesquisadores brasileiros, no qual $53.1 \%$ dos alunos proferiram não seguir alguma religião. Vale lembrar que o contexto religioso, quando presente no dia a dia das pessoas, especialmente entre jovens, representa uma grande influência nas relações familiares, sociais e culturais, implicando positivamente em hábitos e comportamentos saudáveis ${ }^{16}$.

Acerca do risco familiar, o contato próximo com os familiares, principalmente os pais, é considerado fator de proteção para os problemas da adolescência. Os pais e responsáveis devem estar sempre atentos às condutas neste período. É de suma importância a criação de um vínculo entre eles e a passagem de confiança de um para o outro, fazendo com que esse adolescente passe por essa fase com segurança (PeNSE, 2015) ${ }^{5}$. De acordo com dados das edições anteriores (PeNSE 2009 e 2012), os pesquisadores constataram que a supervisão realizada pelos responsáveis dos adolescentes tem resultado em um efeito protetor quanto ao consumo de álcool, tabaco, drogas ilícitas e ao início da vida sexual precoce ${ }^{5}$.

O consumo de drogas lícitas e ilícitas vem sendo uma preocupação mundial, seu uso com frequência causa danos não só psíquicos, mas também biológicos e sociais, além de prejuizos futuros dos usuários. A adolescência é a faixa etária com maior suscetibilidade para experimentar e/ou fazer uso dessas substâncias, 
sendo que alguns fatores podem estar relacionados com a impressão de onipotência, relacionamento familiar e com a procura por novas sensações e experiências ${ }^{17}$.

O primeiro contato com algum tipo de droga, seja ela lícita ou ilícita, decorre especialmente pelos adolescentes terem amigos que usam, o que gera uma certa pressão da parte deles para que haja o consumo e também para ser aceito naquele determinado grupo de amigos ${ }^{18}$.

No que diz respeito ao consumo do tabaco, uma pequena parcela dos adolescentes já fumou pelo menos um cigarro. Estudo realizado em Uruguaiana (RS), também constatou dados significativos ao identificar que 29,3\% dos alunos tentaram fumar cigarro e, destes, apenas $6,8 \%$ relataram ter fumado e referiram fumar a maioria dos/ou todos os dias ${ }^{19}$. No País, de acordo com a PeNSE terceira edição, 30\% dos jovens entre 13 e 15 anos começaram a fumar antes dos 12 anos, visto que a adolescência é o grupo de maior risco para o início do tabagismo, justamente por ser uma fase de descobertas de sensações (2015)5.

O estudo mostra, ainda, que grande parte dos adolescentes já consumiu e/ou consome bebida alcoólica. Dados que corroboram o estudo de pesquisadores brasileiros em instituições privadas, no qual a maioria dos estudantes também já havia consumido bebidas alcoólicas $(85,7 \%)^{20}$. Não obstante, estudo realizado em Porto Velho (RO) constatou que 49,6\% dos adolescentes já consumiram bebidas alcoólicas pelo menos uma vez. sendo que $39,3 \%$ haviam bebido pela primeira vez em casa, juntamente com os familiares e $46,7 \%$ relataram ter o primeiro contato com o álcool por influência dos amigos ${ }^{13}$.

Embora o álcool seja consumido cada vez mais cedo pelos adolescentes ${ }^{13}$, instituiu-se a Lei $n^{0} 13.106$, em 17 de março de 2015, que proíbe e qualifica como crime vender, oferecer, fornecer, entregar e consentir o consumo de bebida alcoólica, ainda que gratuitamente, a menores de dezoito anos de idade em espaços públicos e familiares.

Em relação ao consumo de drogas ilícitas, o número de adolescentes que já fez ou faz uso desse tipo de substância não predominou. Pesquisadores brasileiros desenvolveram uma pesquisa sobre o consumo de álcool em binge, quando há ingestão de cinco doses ou mais em uma única ocasião, e o uso de drogas ilícitas entre adolescentes escolares, na qual foi evidenciado que 10,5\% dos entrevistados já fizeram uso de maconha, droga que se destacou entre as consumidas entre eles, sendo que $6,5 \%$ relataram que já consumiram inalantes e 4,1\%, cocaína²1.

Ao comparar o índice de pensamento e tentativa de suicídio, constatou-se que houve o predomínio em estudantes de escolas privadas. Sabe-se que conflitos psicológicos, problemas familiares, sobrecarga de sentimento de tristeza e raiva, podem estar associados ao pensamento suicida ${ }^{20}$.

Evidencia-se a necessidade da realização de estudos que possam apurar a existência de uma relação entre a excessiva cobrança dos pais e das escolas acerca do rendimento escolar dos adolescentes da rede privada. E, ainda, verificar se a ausência dos responsáveis devido aos seus vínculos empregatícios, é fator capaz de influenciar esses jovens a cometer o ato suicida.

No ambiente e processo escolar, pode-se encontrar inúmeras demandas sociais, dentre as quais a discriminação, o preconceito, a intolerância, o abandono escolar, a violência, a desobediência, o bullying, entre outros $^{22}$.

Ao considerar que a maioria dos estudantes que apresentaram ideação ou tentaram cometer suicídio eram do sexo feminino, faz-se importante mencionar que adolescentes do sexo feminino apresentam maior índice de comprometimentos da saúde psíquica, comportamental e física ${ }^{15}$. Assim como um estudo feito com adolescentes de ensino médio em São Carlos (SP), apontou que dos 30 participantes, sendo 15 meninos e 15 meninas, 12 deles apresentavam ideação suicida, com predomínio do sexo feminino²3. 
Quando avaliados o comportamento sexual e o conhecimento de métodos contraceptivos entre os adolescentes, grande parte dos estudantes ainda não teve a primeira relação sexual. Porém, uma parcela significativa já teve, destacando-se os alunos de escolas públicas.

Pesquisa brasileira revelou que 36,40\% dos alunos entrevistados já tiveram sua primeira relação sexual e, destes, a maioria obteve sua experiência sexual até os 14 anos de idade ${ }^{15}$. De acordo com a PeNSE $(2015)^{5}$. 29,7\% de alunos das escolas públicas e 15,0\% das escolas privadas já tiveram relação sexual alguma vez. O início da vida sexual dos brasileiros normalmente acontece durante a adolescência. Estudos recentes mostram que quanto mais cedo iniciar a vida sexual, as chances de ocasionar algum dano ou prejuízo à saúde do adolescente é maior ${ }^{24}$.

No que tange ao uso de camisinha na primeira relação sexual, o presente estudo mostrou que a maioria dos adolescentes fez uso, resultado que se assemelhou ao de pesquisadores brasileiros, no qual 60\% dos alunos que referiram vida sexual ativa relataram ter usado preservativo na primeira relação sexual. Meninas que possuem parceiro fixo representam o grupo de adolescentes que mais faz uso de preservativo na primeira relação sexual, pois o primeiro contato sexual de parceiros fixos geralmente é planejado, ao contrário de relações ocasionais ${ }^{25}$.

Quanto ao uso de camisinha na última relação sexual, foi evidenciado, na pesquisa, que grande parte dos adolescentes sexualmente ativos relataram fazer uso de preservativo. Pesquisa realizada em Pelotas (RS) evidenciou que, o uso de preservativo na última relação foi feito com mais frequência pelos adolescentes homens e, em contrapartida, cerca de 30,0\% das adolescentes não haviam feito uso de método contraceptivo e $17,9 \%$ não fizeram uso de preservativo ${ }^{24}$.

Sabe-se que os adolescentes possuem conhecimento sobre a importância da prevenção com o uso de preservativos, e de inúmeros fatores que comprometem sua saúde. Contudo, por influência do meio, da vergonha e, por muitas vezes não carregarem consigo o preservativo, preferem não fazer uso ${ }^{10}$.

A idade precoce do início da vida sexual, o uso incorreto de preservativos, a escolha por múltiplos parceiros e o consumo de drogas são fatores que estão fortemente ligados à vulnerabilidade para infecções ${ }^{10}$. Em relação às ISTs, o presente estudo mostrou que somente 2 estudantes de escolas públicas referiram ter contraído algum tipo de infecção. Estudo maranhense identificou que, com relação aos indivíduos vulneráveis às ISTs, 169 (86,7\%) adolescentes responderam que independente da pessoa ter parceiro fixo ou não, se não houver o uso de preservativo nas relações sexuais, as chances de contrair uma IST são muito maiores ${ }^{26}$.

Ao considerar o estado civil dos participantes, a maior parte dos adolescentes que já possuía vida sexual ativa tinha parceiros fixos, divergindo da pesquisa realizada no município de Caxias (MA), em 2017. que avaliou o conhecimento de estudantes de escolas públicas sobre as ISTs. Constatou-se que a maioria dos entrevistados (77,9\%) eram solteiros ${ }^{25}$. É válido destacar que a atividade sexual com múltiplos parceiros eleva as chances de contrair IST.

No que diz respeito ao conhecimento sobre métodos contraceptivos, a maioria dos adolescentes possuía informações sobre tal assunto. Porém, há uma carência maior sobre esse contexto em alunos de escolas públicas em relação aos alunos de escolas privadas. Na pesquisa realizada por pesquisadores brasileiros acerca do conhecimento dos adolescentes sobre IST, AIDS e métodos contraceptivos, 52,1\% dos entrevistados relataram não conhecer outros métodos a não ser a camisinha e pílula anticoncepcional para evitar uma gravidez indesejada ${ }^{27}$.

Tendo em vista que o conhecimento sobre métodos contraceptivos seja de suma importância para prevenção de IST e/ou uma gravidez não planejada, cabe às escolas, pais e aos profissionais na área da saúde 
dispor de conteúdos de educação sexual e reprodutiva para o aumento do conhecimento dos adolescentes sobre tal assunto, visando a diminuição dessa vulnerabilidade.

Diante das diversas vulnerabilidades apresentadas no contexto da adolescência, foram identificadas uma média de 6,23 vulnerabilidades entre os adolescentes, encontrando-se o mínimo de uma vulnerabilidade e o máximo de quinze. Analisou-se que, alunos de escolas públicas apresentaram mais vulnerabilidades em relação aos alunos de escolas privadas. Porém, 100\% dos alunos da rede privada possuíam 5 ou mais vulnerabilidades.

\section{CONCLUSÃO}

Foi possivel constatar que, ambas as escolas apresentaram um elevado número de vulnerabilidades entre os adolescentes, o que não difere do contexto brasileiro, visto que o consumo de drogas, o pensamento e/ou tentativa de suicídio e o comportamento sexual inadequado nesta fase, é nacionalmente considerado um problema de saúde pública.

Assim, salienta-se a importância dos serviços de atenção básica para a promoção e prevenção em saúde, porintermédio de ações de educação em saúde nas escolas voltadas para essa população, juntamente com os responsáveis, com abordagem interdisciplinar, para que ambos possam conhecer as transformações que essa fase ocasiona. Grande parte dos pais acredita que é somente uma fase transitória da vida, não dimensionando os riscos, muitas vezes irreparáveis, aos quais seus filhos poderão estar expostos.

Diante disso, a atuação da enfermagem neste cenário é de grande valia e importância, pois esse profissional possui uma gama de conhecimentos anatômicos e fisiológicos do corpo humano para repassar a esse público e intervir na melhoria e no decréscimo desses índices elevados de vulnerabilidades.

Como limitação do estudo, há de se destacar as possiveis omissões de informações por parte dos alunos e a ausência de seriedade e comprometimento em responder ao questionário adequadamente.

\section{REFERÊNCIAS}

1. Melo CC, Picheli AAWS, Ribeiro KCS. Um estudo comparativo entre o consumo de álcool e tabaco por adolescentes: Fatores de vulnerabilidade e suas consequências. InterScientia. [Internet]. 2016. [Citado em 2019 Out 28]:4(1):21-30.Disponivel em: https://periodicos.unipe.br/ index.php/interscientia/article/view/505/384

2. Amoras BC, Campos AR, Beserra EP. Reflexões sobre vulnerabilidade dos adolescentes a infecções sexualmente transmissiveis. PRACS - Rev Eletr Hum Curso Cien Soc UNIFAP. [Internet]. 2015 [citado em 2019 Jul 15];8(1):163-171. Disponivel em: https://periodicos.unifap.br/ index.php/pracs/article/view/1668/camposv8n1.pdf

3. Costa TS,Capeletti CP, Mello ML, Vieira PR, Brum MD, Krabbe EC, et al. Escola, sexualidade, práticas sexuais e vulnerabilidade para infecções sexualmente transmissiveis (IST). RevInt. [Internet]. 2016. [Citado em 2019 Ago 25]:4(1):75-84. Disponível em: http://www.revistaeletronica.unicruz.edu.br/index.php/eletronica/article/view/4387

4. Ribeiro KCS, Pereira LB, Wiese RB, Silva J. Saldanha AAW. Consumo de álcool e tabaco e associação com outras vulnerabilidades em jovens. Psic, Saúde \& Doenças. [Internet]. 2017. [Citado em 2019 Abr 5]:18(2):348-359. Disponível em: http://www.scielo.mec.pt/scielo. php?script=sci_arttext\&pid=S1645-00862017000200006

5. IBGE. Ministério da Saúde. Pesquisa Nacional de Saúde do Escolar 2015. [Internet] IBGE: Rio de Janeiro. [Citado em 2019 Ago 26].Disponível em: https://biblioteca.ibge.gov.br/visualizacao/livros/liv97870.pdf

6. Lins LS, Silva LAM, Santos RG, Morais TBD, Beltrão TA, Castro JFL. Análise do comportamento sexual de adolescentes. Rev Bras Promoç 
Saúde. [Internet]. 2017. [Citado em 2019 Jun 19]:30(1):47-56. Disponível em: https://www.redalyc.org/pdf/408/40851313007.pdf

7. Neves RG, Wendt A, Flores TR, Costa CS, Costa FS, Rodrigues LT, et al. Simultaneidade de comportamento de risco para infecções sexualmente transmissíveis em adolescentes brasileiros. Epidemiol Serv Saude. [Internet]. 2017. [Citado em 2019 Mar 16]:26(3):443-454. Disponível em: http://www.scielo.br/scielo.php?script=sci_abstract\&pid=\$2237-96222017000300443\&lng=en\&nrm=iso\&tlng=pt

8. Carneiro RF, Silva NC, Alves TA, Albuquerque DO, Brito DC, Oliveira LL. Educação sexual na adolescência: Uma abordagem no contexto escolar. Sanare (Sobral, on-line). [Internet]. 2015. [Citado em 2019 Maio 22]; 14(1):104-108. Disponível em: https://sanare.emnuvens.com.br/ sanare/article/view/617/334

9. Santos CAN, Miranda LESM, Morais RS, Pereira RG, Silva RA, Oliveira FBM. Análise de vulnerabilidades em adolescentes escolares. Rev Cien e Sab (on-line) [Internet]. 2017. [Citado em 2019 Set 15];3(1):338-344. Disponível em: http://www.facema.edu.br/ojs/index.php/ReOnFacema/article/view/141/82

10. Santos JR, Julião CH. O enfrentamento de vulnerabilidade de adolescentes em uma instituição de atenção social. REFACS (on-line) [Internet]. 2016. [Citado em 2019 Mar 18];4(1):33-39. Disponivel em: https://www.redalyc.org/jatsRepo/4979/497950105005/497950105005 pdf

11. Batista, F. A. Comportamento sexual de risco em adolescentes escolares: questionário juventude brasileira fase II [Dissertação]. Santa Maria (RS): Universidade Federal de Santa Maria. 2014 [Citado em 2019 Mar 18] Disponível em:https://repositorio.ufsm.br/handle/1/10334

12. Almeida RAAS, Corrêa RGCF, Rolim ILTP, Hora JM, Linard AG, Coutinho NPS, et al. Conhecimento de adolescentes relacionados às doenças sexualmente transmissiveis e gravidez. Rev Bras Enferm. [Internet]. 2017 [citado em 2019 Jun 9]; 70(5):1087-94. Disponível em:http:// dx.doi.org/10.1590/0034-7167-2016-0531

13. Elicker E, Palazzo LS, Aerts DRGC, Alves GG, Câmara S. Uso de álcool, tabaco e outras drogas por adolescentes escolares de Porto Velho (RO), Brasil. Rev Epidemiol Serv Saúde. [Internet]. 2015. [Citado em 2019 Jun 20]:24(3):399-410. Disponivel em: http://www.scielo.br/scielo. php?script=sci_arttext\&pid=S2237-96222015000300399

14. Instituto Brasileiro de Geografia e Estatística. Diretoria de Pesquisas. Coordenação de Trabalho e Rendimento. Pesquisa Nacional por Amostra de Domicílios Contínua: Características gerais dos domicílios e dos moradores 2018 [Internet]. IBGE: Rio de Janeiro 2019. [Citado em 2019 Ago. 25]. Disponivel em: https://biblioteca.ibge.gov.br/visualizacao/livros/liv101654_informativo.pdf.

15. Mota RS, Gomes NP, Oliveira JF, Campos LM, Araújo GS, Gusmão MEN, et al. Situação sociodemográfica e de saúde em adolescentes escolares segundo o sexo. Rev Bras Enferm. [Internet]. 2019. [Citado em 2019 Set 10]:72(4):1063-9. Disponivel em: http://www.scielo.br/ scielo.php?pid=S0034-71672019000401007\&script=sci_arttext\&tlng=pt.

16. Santos ARM, Oliveira LMFT, Farias JCJ, Silva PPC, Silva EAPC, Freitas CMSM. Associação entre prática religiosa e comportamentos de risco à saúde em adolescentes de Pernambuco, Brasil. Rev Bras Ativ Fís Saúde. [Internet]. 2015. [Citado em 2019 Ago 11];20(3):284-296. Disponivel em: http://rbafs.org.br/RBAFS/article/view/5046/4467

17. Medeiros KT, Maciel SC, Sousa PF, Souza FMT, Dias CCV. Representações sociais do uso e abuso de drogas entre familiares de usuários. Rev Psicol Estud. [Internet]. 2013. [Citado em 2019 Out 24];18(2): 269-279. Disponível em:http://www.scielo.br/pdf/pe/v18n2/a08v18n2.pdf

18. Nascimento MO, Avallone DM. Prevalência do uso de drogas entre adolescentes nos diferentes turnos escolares. Rev. Adolesc. Saúde. [Internet]. 2013. [Citado em 2019 Jul 10];10(4):41-49. Disponivel em: http://adolescenciaesaude.com/detalhe_artigo.asp?id=424

19.Pereira MU, Oliano VJ, Aranda CS, Mallol J, Solé D. Prevalência e fatores associados ao tabagismo entre adolescentes. Rev J Pediatr. (Rio J.).[Internet]. 2017. [Citado em 2019 Mar17];93(3):230-7. Disponivel em: http://www.scielo.br/scielo.php?pid=S002175572017000300230\&script=sci_arttext\&tlng=pt

20. Zeferino AM, Kalinoski A, Teixeira GT, Costa LD, Zonta FNS. Fatores de risco em adolescentes de instituições de ensino privadas de um município do Paraná. Rev Cien Cuid Saude[Internet]. 2019. [Citado em 2019 Out 29];18(3). Disponivel em: http://periodicos.uem.br/ojs/index. $\mathrm{php} /$ CiencCuidSaude/article/view/45853

21. Raposo ACS, Costa AC, Valança P. Colares V, Franca C. O consumo de álcool em binge e o uso de drogas ilícitas entre adolescentes escolares. Rev Aten Saúde. [Internet]. 2016. [Citado em 2019 Mar 23];14(48):27-31. Disponivel em: https://seer.uscs.edu.br/index.php/revista_ciencias_saude/article/view/3544

22. Silva LGM, Ferreira TJ. O papel da escola e suas demandas. Rev Periódico Científico Projeção e Docência. [Internet]. 2014. [Citado em 2019 Set 16];5(2):6-23.Disponivel em:http://revista.faculdadeprojecao.edu.br/index.php/Projecao3/article/view/415/372

23. Luca LAF, Costa DAO, Souza RM. Ideação Suicida em Adolescentes de 15 a 18 Anos Estudantes do Ensino Médio da Microrregião de São Carlos/SP. Brazilian Journal of Forensic Sciences, Medical Law and Bioethics. [Internet]. 2017. [Citado em 2019 Ago17]:6(4):475-484. Disponivel em: http://www.ipebj.com.br/forensicjournal/edicoes?volume=6\&numero=4\&artigo=264

24. Gonçalves H, Machado EC, Soares ALG, Figuera FAC, Seering LM, Mesenburg MA, et al. Início da vida sexual entre adolescentes (10 a 14 anos) e comportamentos em saúde. Rev Bras Epidemiol. [Internet]. 2015. [Citado em 2019 Jul 15];18(1):1-18. Disponível em: http://www. scielo.br/pdf/rbepid/v18n1/1415-790X-rbepid-18-01-00025.pdf 
25. Campos TE, Martins RA. Relação entre conduta, conhecimento sexual e uso de preservativo entre alunos e professores do Ensino Médio. Rev Adolesc Saúde (On-line). 2017. [Citado em 2019 Jul 18]:14(1):37-44. Disponível: http://www.adolescenciaesaude.com/detalhe_artigo. asp?id=634

26. Carvalho O, Pinto RGS, Santos MS. Conhecimento sobre as infecções sexualmente transmissiveis por estudantes adolescentes de escolas públicas. Rev Adolesc Saúde (On-line). 2018. [Citado em 2019 set 11]:15(1):7-17. Disponível em: http://www.adolescenciaesaude.com/ detalhe_artigo.asp?id=703

27. Miranda AAM, Silva CGO, Thimoteo GM, Assis LF, Del'duca A, Carvalho AR, et al. Conhecimentos acerca de DST/AIDS e métodos contraceptivos dos discentes dos cursos técnicos integrados do IF sudeste MG - campus Juiz de Fora, Brasil. Multiverso. [Internet]. 2016. [Citado em 2019 Out 27];1(1):25-36. Disponivel em: http://webcache.googleusercontent.com/search?q=cache:http://periodicos.jf.ifsudestemg.edu.br/ multiverso/article/viewFile/4/6 